\title{
Fine structure of the confining string in an analytically solvable 3D model
}

\author{
Davide Vadacchino*, Michele Caselle, Roberto Pellegrini \\ Dipartimento di Fisica Teorica, Università di Torino \\ and Istituto Nazionale di Fisica Nucleare, sezione di Torino, \\ Via Pietro Giuria 1, I-10125 Torino, Italy \\ E-mail: vadacchi@to.infn.it, caselledto.infn.it, ropelleg@to.infn.it
}

Marco Panero

Instituto de Física Téorica UAM/CSIC, Universidad Autónoma de Madrid

Calle Nicolás Cabrera 13-15, Cantoblanco E-28049 Madrid, Spain

E-mail: marco.panero@inv.uam.es

In $\mathrm{U}(1)$ lattice gauge theory in three spacetime dimensions, confinement can be analytically shown to persist at all values of the coupling. Furthermore, the explicit predictions for the dependence of string tension $\sigma$ and mass gap $m_{0}$ on the coupling allow one to tune their ratio at will. These features, and the possibility of obtaining high-precision numerical results via an exact duality map to a spin model, make this theory an ideal laboratory to test the effective string description of confining flux tubes. In this contribution, we discuss our investigation of next-toleading-order corrections to the confining potential and of the finite-temperature behavior of the flux tube width. Our data provide a very stringent test of the theoretical predictions for these quantities and allow to test their dependence on the $m_{0} / \sqrt{\sigma}$ ratio.

IFT-UAM/CSIC-13-104

31st International Symposium on Lattice Field Theory LATTICE 2013

July 29, 2013

Mainz, Germany

${ }^{*}$ Speaker. 


\section{Introduction}

One of the most interesting recent results on the effective string description of Lattice Gauge Theories (LGTs) is the proof of universality of the first few terms of the effective action. This action can be written as a low-energy expansion in the number of derivatives of the transverse degrees of freedom of the string. The first term of this expansion is simply the $2 \mathrm{D}$ massless free field theory [1]

$$
S[X]=S_{c l}+\frac{\sigma}{2} \int d^{2} \xi\left(\partial_{\alpha} X \cdot \partial^{\alpha} X\right)+\ldots,
$$

where the classical action $S_{c l}$ describes the usual perimeter-area term, $X$ denotes the two-dimensional bosonic fields $X_{i}\left(\xi_{1}, \xi_{2}\right)$, with $i=1,2, \ldots, D-2$, describing the transverse displacements of the string with respect the configuration of minimal action and $\xi_{1}, \xi_{2}$ are the coordinates on the worldsheet. The next few terms beyond the free 2D bosonic theory can be parametrized as follows [2]

$$
S=S_{c l}+\frac{\sigma}{2} \int d^{2} \xi\left[\partial_{\alpha} X \cdot \partial^{\alpha} X+c_{2}\left(\partial_{\alpha} X \cdot \partial^{\alpha} X\right)^{2}+c_{3}\left(\partial_{\alpha} X \cdot \partial_{\beta} X\right)^{2}+\ldots\right],
$$

The coefficients $c_{i}$ must satisfy a set of constraints. These constraints were first obtained by comparing the string partition function in different channels ("open-closed string duality") [2, 3]. However it was later realized [4-6] that they are a direct consequence of the Lorentz symmetry of the underlying Yang-Mills theory. Indeed, even if the complete $S O(1, D-1)$ invariance is broken by the classical configuration around which we expand, the effective action should still respect this symmetry through a non-linear realization in terms of the transverse fields $X_{i}$. In this way it was shown [5] that the terms with only first derivatives coincide with the Nambu-Goto action to all orders in the derivative expansion. The first allowed correction to the Nambu-Goto action turns out to be the the six-derivative term [3]

$$
c_{4}\left(\partial_{\alpha} \partial_{\beta} X \cdot \partial^{\alpha} \partial^{\beta} X\right)\left(\partial_{\gamma} X \cdot \partial^{\gamma} X\right)
$$

with arbitrary coefficient $c_{4}$; however this term is non-trivial only when $D>3$. The fact that the first deviations from the Nambu-Goto string are of high order, especially in $D=3$, explains why in early Monte Carlo calculations [7, 8] good agreement with the Nambu-Goto string was observed (for an updated review of Monte Carlo results see [9-11]). This agreement led to the hope to be able to identify, via numerical simulations, some non trivial "stringy" feature of the interquark potential. For instance in $D=3$ the first non-trivial deviation of the Nambu-Goto action occurs at the level of eight-derivative terms and it was recently shown [12], that this term is proportional to the squared curvature of the induced metric on the world-sheet.

However, in the last few years, thanks to the remarkable improvement in the accuracy of simulations, systematic deviations from the expected universal behaviour were observed well below the eight-derivatives term mentioned above [13-15]. These deviations were observed both in the excited string states in (3+1)-dimensional SU(N) LGTs [13] and in the ground state (i.e. the interquark potential) in the 3D Ising gauge model. In this last case deviation were observed both in the torus (interface) [14] and in the cylinder (Polyakov loop correlators) [15] geometries.

A possible reason for these deviations could be the presence of massive modes in the world sheet due to the coupling of the effective string to glueball states. If this is the case then one could 
try to model these massive modes and include them in the fits. The main goal of this proceeding is to test this picture in the case of compact U(1) LGT in three dimensions. This is a perfect laboratory for this type of analysis since in this model the $m_{0} / \sqrt{\sigma}$ ratio is not fixed but can be tuned as a function of the coupling. Thus by tuning $\beta$ we can compare the ability of the effective string to fit the interquark potential when the $m_{0} / \sqrt{\sigma}$ takes a value comparable with those of the Ising or SU(N) LGTs (i.e. $m_{0} / \sqrt{\sigma} \sim 3$ ) and for a much lower value $m_{0} / \sqrt{\sigma} \sim 1$. As we shall see for $m_{0} / \sqrt{\sigma} \sim 1$ the effective string predictions strongly disagree with the numerical data.

\section{The $U(1)$ model in $2+1$ dimensions}

The U(1) lattice gauge model in $D=2+1$ can be solved in the semiclassical approximation $[16,17]$. It can be shown that the model is always confining and that in the $\beta \gg 1$ limit it flows toward a theory of free massive scalars. In this limit the mass of the lightest glueball and the string tension should behave as

$$
m_{0}=c_{0} \sqrt{8 \pi^{2} \beta} e^{-\pi^{2} \beta v(0)}, \quad \sigma \geq \frac{c_{\sigma}}{\sqrt{2 \pi^{2} \beta}} e^{-\pi^{2} \beta v(0)},
$$

where in the semiclassical approximation $c_{0}=1$ and $c_{\sigma}=8$. Previous numerical studies [18] (which we confirm with our simulations) showed that the string tension saturates the above bound and that both constants are affected by the semiclassical approximation and change their values in the continuum limit. Despite this difference, both $m_{0}$ and $\sigma$ remain strictly positive in the whole range of values of $\beta$, the model is thus always confining. The point in using such lattice model at finite spacing is that, while in general for confining gauge theories the $m_{0} / \sqrt{\sigma}$ ratio is fixed, in this model we have:

$$
\frac{m_{0}}{\sqrt{\sigma}}=\frac{c_{0}}{\sqrt{c_{\sigma}}} 2 \pi(2 \pi \beta)^{3 / 4} e^{-\pi^{2} v(0) \beta / 2}
$$

so that we can tune the ratio to any chosen value by tuning $\beta$.

Since the model is invariant under an Abelian gauge symmetry, one can easily perform a duality transformation and obtain a simple spin model with global $\mathbb{Z}$ symmetry (note that, in $D=$ $3+1$ dimensions, the same transformation leads to a model with local $\mathbb{Z}$ symmetry [19-23]). The dual formulation has many advantages over the original one. First of all, from the computational point of view, the model is much easier and faster to simulate, since we deal with a spin model. Moreover, the presence of a static $Q \bar{Q}$ pair at a distance $R$ in the form of Polyakov loops can be easily included in the partition function that becomes, in that case

$$
Z_{R}=\sum_{\left\{{ }^{\star} l=-\infty\right.}^{\infty} \prod_{{ }^{\star} C_{1}} \mathrm{I}_{\left|\mathrm{d}^{\star} l+^{\star} n\right|}(\beta)
$$

where $n$ is a 2-form which must be non-vanishing on an arbitrary surface bounded by the two loops; without loss of generality, we choose the one of minimal area.

\section{Results}

We performed our simulations on the dual model, using a single site metropolis algorithm, at three different values of the coupling, on $(64 a)^{2} \times L$ lattices, with $L$ the lattice size in the Euclidean 
time direction. We chose two values $L=16 a$ and $L=64 a$ and evaluated the interquark potential for all values of the interquark distance $R$ in the range $8 a \leq R \leq 32 a$ so as to have a wide range of values of $R$, such that $2 R<L$ and $2 R>L$ for $L=64 a$ and $L=16 a$ respectively. As we shall see below in these two limits the effective string predictions for the interquark potential simplify and fitting the simulation results becomes much simpler. The values of the coupling were chosen so that the $m_{0} / \sqrt{\sigma}$ ratio ranged from $\sim 2.5$ for $\beta=1.7$ to $\sim 0.7$ for $\beta=2.75$. Details on the simulation setting are reported in tab. 1 .

The simulations were performed on the dual model using the snake algorithm [24] with a hierarchical update algorithm [25] to decorrelate data.

Following $[8,25]$ we computed the quantity

$$
Q(R)=-\frac{a}{L} \log \left[\frac{G(R+a)}{G(R)}\right]=a[V(R)-V(R+a)]
$$

in which the perimeter and costant terms of the interquark potential cancel. Setting in eq. (1.2) the values $c_{2}=\frac{1}{8}$ and $c_{3}=-\frac{1}{4}$ prescribed by Lorentz invariance, we find at next-to-leading order [8],

$$
Q(R)= \begin{cases}\sigma a^{2}+\frac{\pi a^{2}}{24 R(R+a)}+\frac{\pi^{2} a^{2}}{1152 \sigma} \frac{2 R^{2}+2 a R+a^{2}}{R^{3}(R+a)^{3}}+\cdots & 2 R \ll L \\ \sigma a^{2}\left(1-\frac{\pi}{6 L^{2} \sigma}-\frac{\pi^{2}}{72 \sigma^{2} L^{4}}+\cdots\right)+\frac{a^{2}}{2 L R}-\frac{a^{2}}{8 \sigma L^{2} R^{2}}+\cdots & 2 R \gg L\end{cases}
$$

We fitted the data obtained in the simulations with eqs. 3.2 keeping $\sigma a^{2}$ as the only free parameter and used the reduced $\chi^{2}$ to estimate the quality of the various fits. We performed two types of fits. In the first we kept for all values of $\beta$ exactly the same number of degrees of freedom in the fit, fitting for both $L=16 a$ and $L=64 a$ all the data in the range $12 a \leq R \leq 32 a$. In the second we fitted the data in the range $R_{\min } \leq R \leq 32 a$ choosing different thresholds $R_{\min }$ so as to keep the adimensional ratio $\sqrt{\sigma} R_{\min } \sim 2$ for each value of $\beta$. This second choice decreases the number of degrees of freedom for the larger values of $\beta$ but eliminates the bias due to the difference in the value of $1 /(a \sqrt{\sigma})$ in the three samples. Results are reported in tabs. 2 and 3, respectively. We also plot in fig. 1 and fig. 2 the result of the fit in the two limiting cases $\beta=1.7$ and $\beta=2.75$.

\begin{tabular}{|c|c|c|}
\hline$\beta$ & $m_{0} / \sqrt{\sigma}$ & $\sigma a^{2}$ \\
\hline 1.7 & $2.5(1)$ & $0.1227(1)$ \\
\hline 2.2 & $1.6(2)$ & $0.0273(1)$ \\
\hline 2.75 & $0.7(1)$ & $0.0070(1)$ \\
\hline
\end{tabular}

Table 1: Values of $m_{0} / \sqrt{\sigma}$ and $\sigma a^{2}$ for the three values of $\beta$. While the dependence on $\beta$ follows the semiclassical prediction, the constant in front of $\sigma$ and $m_{0}$ differ from the semiclassical ones

Looking at tabs. 2 and 3 and at figs. 1 and 2 we see that for $\beta=1.7$ (for which $m_{0} / \sqrt{\sigma} \sim 2.5$ ) the effective string describes very precisely the data while for $\beta=2.75$ (for which $m_{0} / \sqrt{\sigma} \sim 0.7$ ) there is clear disagreement. In this second case even the first order effective string correction, the Lüscher term, is excluded by the data. This behaviour is in striking disagreement with the results of almost all the numerical experiments performed in the last few years in other lattice gauge theories where the Lüscher term was always found to agree with the data for large enough values of $R$.

These results show that the ability of the effective string to describe the interquark potential is strongly related to the ratio $m_{0} / \sqrt{\sigma}$, and worsens as the lowest glueball mass approaches $\sqrt{\sigma}$. This 

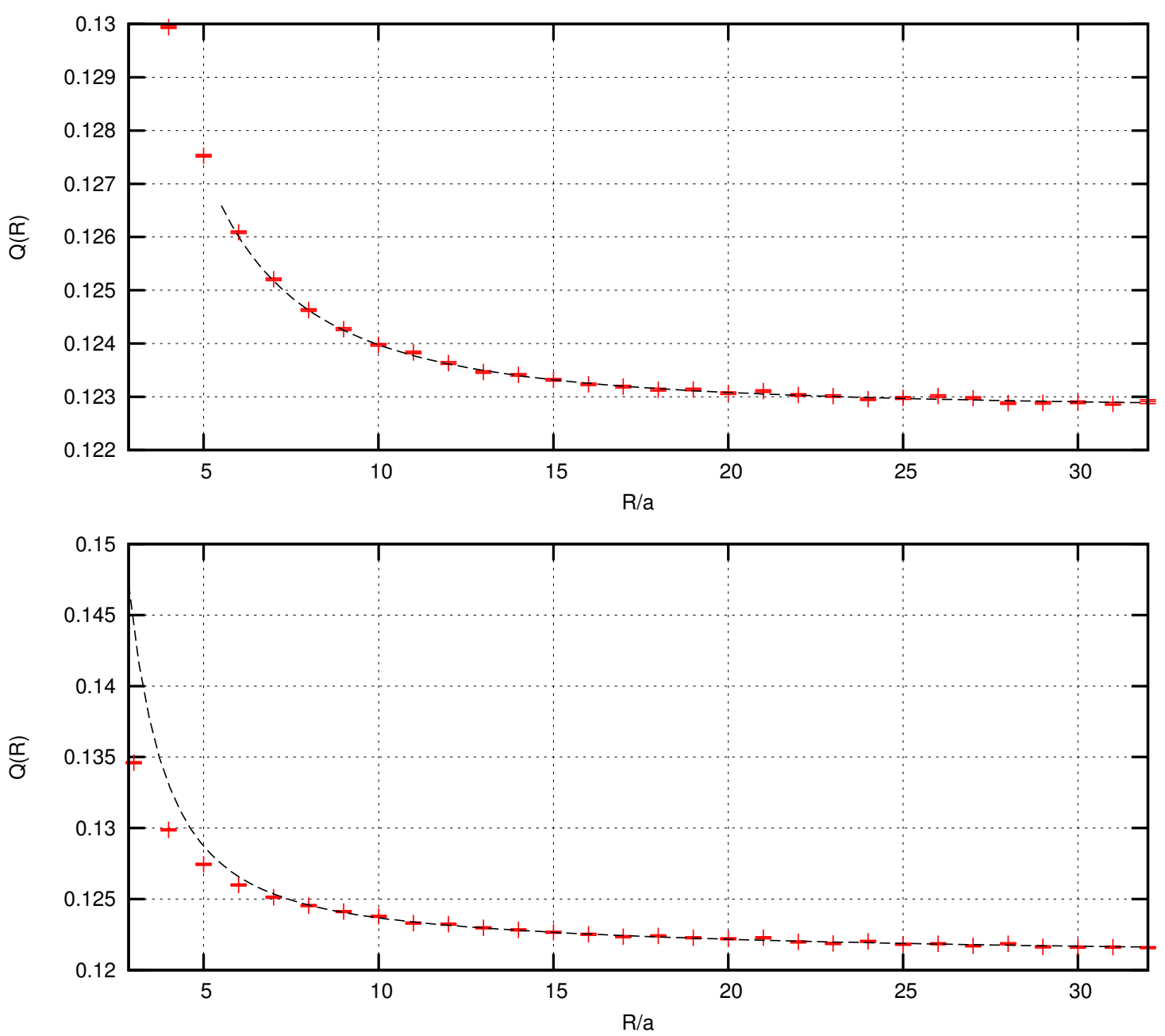

Figure 1: Results from simulations at $\beta=1 /\left(a e^{2}\right)=1.7$. For this coupling, $m_{0} / \sqrt{\sigma} \simeq 2.5$ and $1 /(a \sqrt{\sigma}) \simeq$ 3. The values of $L$ and $R$ are chosen so that $R<L / 2$ for the plot in the top panel, $R>L / 2$ for the one at the bottom. The curves correspond to the best fit results obtained with eqs. (3.2) setting $R_{\min }=12 a$.

preliminary test suggests that indeed the emission of glueballs can masks the stringy behaviour of the system and could be the ultimate reason of the discrepancies observed in refs. [13-15]. Similarly to other 3D models [26], the 3D U(1) model could be a very powerful tool to study the interplay between effective strings and glueballs.

Acknowledgments This work is supported by the Spanish MINECO's "Centro de Excelencia Severo Ochoa" programme under grant SEV-2012-0249. 

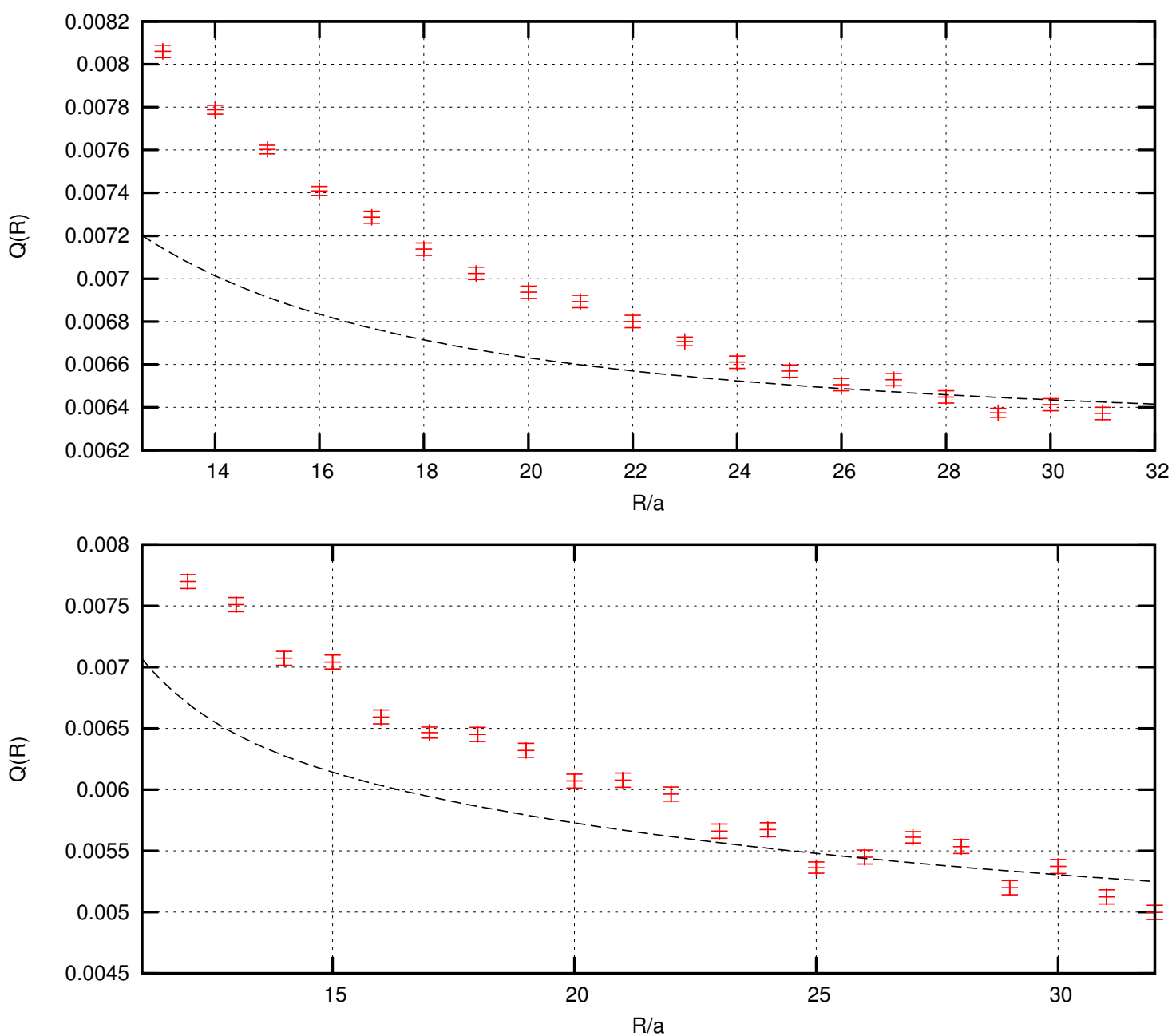

Figure 2: Same as in fig. 1, but for $\beta=2.75$ (for which $m_{0} / \sqrt{\sigma} \simeq 0.7$ and $1 /(a \sqrt{\sigma}) \simeq 12$ ) and $R_{\min }=24 a$.

\begin{tabular}{|c||c|c|c|c|}
\hline$\beta$ & $L=64 a$ & $L=16 a$ & $R_{\min } / a$ & d.o.f. \\
\hline 1.7 & 1.1 & 1.2 & 12 & 20 \\
\hline 2.2 & 2.2 & 9.9 & 12 & 20 \\
\hline 2.75 & 47.4 & 178.6 & 12 & 20 \\
\hline
\end{tabular}

Table 2: Values of $\chi_{r}^{2}$ obtained by comparing the values $Q(R)$ obtained in lattice simulations with the prediction of effective string theory at NLO, for $R \geq R_{\text {min }}$.

\begin{tabular}{|c||c|c|c|c|}
\hline$\beta$ & $L=64 a$ & $L=16 a$ & $R_{\min } / a$ & d.o.f. \\
\hline 1.7 & 1.1 & 1.2 & 8 & 24 \\
\hline 2.2 & 1.8 & 2.2 & 16 & 16 \\
\hline 2.75 & 9.4 & 5.1 & 24 & 8 \\
\hline
\end{tabular}

Table 3: Same as tab. 2 but for different values of $R_{\min }$ for the different values of $\beta$. 


\section{References}

[1] M. Lüscher, K. Symanzik and P. Weisz, Nucl. Phys. B173, 365 (1980).

[2] M. Lüscher and P. Weisz, JHEP 07, 014 (2004), [arXiv: hep-th/ 0406205 ] .

[3] O. Aharony and E. Karzbrun, JHEP 06, 012 (2009), [arXiv:0903.1927 [hep-th] ].

[4] H. B. Meyer, JHEP 05, 066 (2006), [arXiv: hep-th/ 0602281 ] .

[5] O. Aharony and M. Field, JHEP 1101, 065 (2011), [arXiv:1008.2636 [hep-th ] ] .

[6] F. Gliozzi, Phys. Rev. D84, 027702 (2011), [arXiv:1103.5377 [hep-th] ].

[7] M. Caselle et al., Nucl. Phys. B432, 590 (1994), [arXiv: hep-lat/9407002 ] .

[8] M. Caselle, M. Hasenbusch and M. Panero, JHEP 03, 026 (2005), [arXiv: hep-lat/ 0501027 ] .

[9] B. Lucini and M. Panero, Phys. Rept. 526 (2013) 93, [arXiv:1210.4997 [hep-th] ] .

[10] M. Panero, PoS (LATTICE 2012) 010, [arXiv:1210.5510 [hep-lat]].

[11] B. Lucini and M. Panero, arXiv:1309.3638 [hep-th] .

[12] O. Aharony and M. Dodelson, JHEP 1202 (2012) 008, [arXiv:1111.5758 [hep-th] ] .

[13] A. Athenodorou, B. Bringoltz and M. Teper, JHEP 02, 030 (2011), [arXiv: 1007.4720 [hep-lat] ].

[14] M. Caselle, M. Hasenbusch and M. Panero, JHEP 0709 (2007) 117, [arXiv: 0707.0055 [hep-lat] ].

[15] M. Caselle and M. Zago, Eur. Phys. J. C71 (2011) 1658, [arXiv:1012.1254 [hep-lat] ] .

[16] A. M. Polyakov, Nucl. Phys. B120 (1977) 429.

[17] M. Göpfert and G. Mack, Commun. Math. Phys. 82 (1981) 545.

[18] M. Loan et al., Phys. Rev. D68 (2003) 034504, [arXiv:hep-lat/0209159].

[19] M. Zach, M. Faber and P. Skala, Phys. Rev. D57 (1998) 123, [arXiv: hep-lat/9705019].

[20] M. Panero, JHEP 0505 (2005) 066, [arXiv: hep-lat/ 0503024 ] .

[21] M. Panero, Nucl. Phys. Proc. Suppl. 140 (2005) 665, [arXiv:hep-lat/0408002] .

[22] E. Cobanera, G. Ortiz and Z. Nussinov, Adv. Phys. 60 (2011) 679, [arXiv: 1103.2776 [cond-mat.stat-mech] ].

[23] Y. D. Mercado, C. Gattringer and A. Schmidt, Phys. Rev. Lett. 111 (2013) 141601, [arXiv:1307.6120 [hep-lat]].

[24] Ph. de Forcrand, M. D'Elia and M. Pepe, Phys. Rev. Lett. 86 (2001) 1438, [arXiv:hep-lat/0007034].

[25] M. Caselle, M. Hasenbusch and M. Panero, JHEP 0301 (2003) 057, [arXiv:hep-lat/0211012].

[26] A. Athenodorou, B. Bringoltz and M. Teper, JHEP 1105 (2011) 042, [ arXiv: 1103.5854 [hep-lat] ]. 